\title{
Planetoid string solutions in $3+1$ axisymmetric spacetimes
}

\author{
H.J. de Vega \\ Laboratoire de Physique Théorique et Hautes Énergies \\ Universités Paris VI-VII - Laboratoire associé au CNRS no 280 \\ Tour 16, 1er. ét., 4, Place Jussieu, 75252 Paris Cedex 05 \\ FRANCE \\ I.L. Egusquizat \\ Fisika Teorikoaren Saila \\ Euskal Herriko Unibertsitatea \\ 644 P.K. - 48080 BILBAO \\ SPAIN
}

(May 31, 2021)

\begin{abstract}
The string propagation equations in axisymmetric spacetimes are exactly solved by quadratures for a planetoid Ansatz. This is a straight non-oscillating string, radially disposed, which rotates uniformly around the symmetry axis of the spacetime. In Schwarzschild black holes, the string stays outside the horizon pointing towards the origin. In de Sitter spacetime the planetoid rotates around its center. We quantize semiclassically these solutions and analyze the spin $/\left(\right.$ mass $\left.^{2}\right)$ (Regge) relation for the planetoids, which turns out to be non-linear.
\end{abstract}

11.25.-w, 04.70.-s, 98.80.Cq 


\section{INTRODUCTION AND MOTIVATIONS}

The systematic investigation of strings in curved spacetimes started in [1] has uncovered a variety of new physical phenomena (see [2,3] for a general review). These results are relevant both for fundamental (quantum) strings and for cosmic strings, which behave in an essentially classical way.

The study of classical and semiclassical strings in curved backgrounds will provide and is indeed providing us with a better comprehension of what a consistent string theory and gravity theory entail. In this context we place the present paper, which continues the line of research set by [1].

Among the heretofore existing analysis of the motion of classical strings in gravitational backgrounds a special place is to be granted to exact solutions, usually obtained by means of separable ansätze (non-separable exact solutions were systematically constructed for de Sitter spacetime [4]). Such are the circular string ansatz [5,6], which for stationary axially symmetric spacetimes reduces the nonlinear equations of string motion to an equivalent one-dimensional dynamical system [7], or the stationary string ansatz [8].

In this paper we examine a different ansatz, which we have called the planetoid ansatz, in stationary axisymmetric $3+1$ spacetime backgrounds. The planetoid solutions are straight non-oscillating string solutions that rotate uniformly around the symmetry axis of the spacetime. In Schwarzschild black holes, they are permanently pointing towards $r=0$ while they rotate outside the horizon. In de Sitter spacetime the planetoid rotates around its center.

We call our ansatz planetoid since it generalizes to strings the bounded circular orbits of point particles in such spacetimes. In the case of the Schwarzschild geometry the planetoid string solutions presented here are generalization of the circular orbits of planets.

We will show how our planetoid ansatz produces either orbiting strings with bounded world-sheet and length or strings of unbounded length. The main competing physical forces in the context of this ansatz are the attraction of gravity, the centrifugal force, and the string tension. The combination of these three causes in different proportions produce different effects, as we will now see.

It should be noted that the effects mentioned take place even when the gravitational field acting on the string is not strong. They are due to the non local character of the string.

We quantize semiclassically the planetoid string solutions using the WKB method adapted to periodic string solutions [9]. We obtain in this way their masses as a function of the angular momentum. Such relations are non-linear and can be considered as a (generalized) Regge trajectory [See figs. 1 and 2].

\section{EQUATORIAL PLANETOID ANSATZ}

\section{A. The ansatz and the string equations of motion}

We consider our classical strings propagating in $3+1$ dimensional stationary axisymmetric spacetime. For simplicity, we restrict in this paper to strings propagating in the equatorial plane $z=0$. We can thus restrict ourselves to the $2+1$ metric with line element of the form

$$
\mathrm{d} s^{2}=g_{t t}(r) \mathrm{d} t^{2}+g_{r r}(r) \mathrm{d} r^{2}+2 g_{t \phi}(r) \mathrm{d} t \mathrm{~d} \phi+g_{\phi \phi}(r) \mathrm{d} \phi^{2} .
$$


Let $\tau$ and $\sigma$ be the time-like and space-like world-sheet coordinate respectively in the conformal gauge. Under the ansatz

$$
\begin{aligned}
t & =t_{0}+\alpha \tau, \\
\phi & =\phi_{0}+\beta \tau, \\
r & =r(\sigma),
\end{aligned}
$$

the equations of motion for a string in this background are given by the following onedimensional equivalent system:

$$
\begin{gathered}
\left(\frac{\mathrm{d} r}{\mathrm{~d} \sigma}\right)^{2}+g^{r r}\left[\alpha^{2} g_{t t}+2 \alpha \beta g_{t \phi}+\beta^{2} g_{\phi \phi}\right]= \\
\left(\frac{\mathrm{d} r}{\mathrm{~d} \sigma}\right)^{2}+V(r)=0 .
\end{gathered}
$$

The function $r(\sigma)$ will then be given by the zero energy motion in $\sigma$ "time" of $r$ under the potential $V(r)=\alpha^{2} g^{r r}\left[g_{t t}+2 \lambda g_{t \phi}+\lambda^{2} g_{\phi \phi}\right]$, with $\lambda=\beta / \alpha$.

Quite obviously, the movement of the string will be periodic. The physical period $T$ in coordinate time $t$ relates to $\lambda$ through

$$
T=\frac{2 \pi}{\lambda}
$$

It will prove useful to introduce the 'physical' potential

$$
\tilde{V}(r)=V(r) / \alpha^{2}=g^{r r}\left[g_{t t}+\frac{4 \pi}{T} g_{t \phi}+\frac{4 \pi^{2}}{T^{2}} g_{\phi \phi}\right],
$$

since it only depends on the physical parameter $T$.

The boundary conditions for open strings, namely, $\partial X_{\mu} / \partial \sigma=0$ at the ends of the string, are naturally fulfilled by this ansatz.

Following [10], we see that this is the only ansatz that separates variables, lets strings be dynamical, and respects the open string boundary conditions, when $r=r(\sigma)$ is chosen.

Note also that this ansatz differs from the circular string ansatz (i.e., $t=t(\tau), \phi=\phi_{0}+\nu \sigma$, $r=r(\tau))$ in the dependence of $r$ in the space-like world-sheet (conformal) coordinate and in the form of the equivalent one-dimensional energy equation, which for this later case reads $\dot{r}^{2}+g^{r r}\left[\mu^{2} g^{t t}+\nu^{2} g_{\phi \phi}\right]$, where the dot stands for the derivative with respect to $\tau$.

The invariant size of the planetoid string is given by the substitution of the ansatz in the line element:

$$
\mathrm{d} s^{2}=g_{r r}\left(\frac{\mathrm{d} r}{\mathrm{~d} \sigma}\right)^{2}\left(-\mathrm{d} \tau^{2}+\mathrm{d} \sigma^{2}\right)
$$




\section{B. Energy and angular momentum}

It is well known that the definition of a stress-energy tensor for an extended object in general relativity is no mean task [11]. In the case at hand, however, there exists a favored time coordinate, for which a Killing vector exists $(\partial / \partial t)$. This allows us to define clearly what is meant as energy, [11]: $\mathcal{E}=\alpha / \alpha^{\prime}$.

Similarly, the existence of the Killing vector $\partial / \partial \phi$, associated with the rotational symmetry, allows for the definition of an angular momentum about the axis. In particular, this is performed as follows: the function $\phi(\sigma, \tau)$ appears in the string Lagrangian only through its derivatives, whence the conserved world-sheet current is obtained by Noether's theorem

$$
J_{\mu}=\frac{2}{\pi \alpha^{\prime}}\left[g_{t \phi} \partial_{\mu} t+g_{\phi \phi} \partial_{\mu} \phi\right] .
$$

The integration of this current provides us with the string angular momentum $J$,

$$
J \equiv \int J_{\tau} d \sigma=\frac{2}{\pi \alpha^{\prime}} \int_{r_{\min }}^{r_{\max }} \mathrm{d} r \frac{g_{t \phi}+\frac{2 \pi}{T} g_{\phi \phi}}{\sqrt{-\tilde{V}(r)}}
$$

where we used eq.(2.3) and $r_{\min }$ and $r_{\max }$ denote the minimum and maximum radius reached by the string, respectively.

\section{General expressions and quantization condition}

We will collect here the expressions for the physical string magnitudes: angular momentum $J$, classical action for solutions $S_{\mathrm{cl}}$, mass $m$, and reduced action $W(m)$. The mass will be defined as $m:=-\mathrm{d} S_{\mathrm{cl}} / \mathrm{d} T$, with $T$ the period. The reduced action [9] is thus obtained as $W(m)=m T(m)+S_{\mathrm{cl}}(T(m))$. The quantization condition will read $W(m)=2 \pi n$ (in units with $\hbar=1)$.

For the case at hand, closed expressions in terms of quadratures can be obtained for all these quantities, as follows:

$$
\begin{aligned}
S_{\mathrm{cl}}(T) & =-\frac{2 T}{\pi \alpha^{\prime}} \int_{r_{\min }}^{r_{\max }} \mathrm{d} r g_{r r} \sqrt{-\tilde{V}(r)}, \\
W & =\frac{4}{T \alpha^{\prime}} \int_{r_{\min }}^{r_{\max }} \mathrm{d} r \frac{T g_{t \phi}+2 \pi g_{\phi \phi}}{\sqrt{-\tilde{V}(r)}}, \\
m & =\frac{W-S_{\mathrm{cl}}}{T} \\
J & =\frac{W}{2 \pi} .
\end{aligned}
$$

As is immediately obvious from these expressions, it is not necessary to have the solution $r(\sigma)$ in a closed form for the quantities indicated to be evaluated, and in what follows we will not use the explicit expressions for $r=r(\sigma)$, which, after all, is dependent on the parametrization of the world-sheet. It should be noted that the previously mentioned quantization condition $[W(m)=2 \pi n]$ is equivalent for this class of solutions to $J=n$. 
This should be interpreted as a consistency check of the semiclassical quantization being performed.

The invariant string length at a fixed time $t$ follows from eq.(2.4)

$$
s=\int_{r_{\min }}^{r_{\max }} \mathrm{d} r \sqrt{g_{r r}} .
$$

\section{EXPLICIT SOLUTIONS AND THEIR ANALYSIS}

\section{A. Minkowski spacetime}

In order to improve our understanding of the physical meaning of the solutions being examined, let us take the easy Minkowski case, for which $g_{t t}=-1, g_{r r}=1, g_{t \phi}=0$, and $g_{\phi \phi}=r^{2}$. Equation (2.3) then becomes

$$
\left(\frac{d r}{d \sigma}\right)^{2}+\lambda^{2} r^{2}-1=0
$$

The solution is immediate:

$$
r=\frac{T}{2 \pi}\left|\cos \left(\frac{2 \pi}{T} \sigma\right)\right|,
$$

where $0 \leq \sigma \leq T / 2$. In cartesian coordinates,

$$
\begin{aligned}
& x=\frac{T}{2 \pi} \cos \left(\frac{2 \pi \tau}{T}\right) \cos \left(\frac{2 \pi \sigma}{T}\right) \\
& y=\frac{T}{2 \pi} \sin \left(\frac{2 \pi \tau}{T}\right) \cos \left(\frac{2 \pi \sigma}{T}\right) .
\end{aligned}
$$

It is easy to see that this is a string of length $T / \pi$ rotating around its middle point which coincides with the origin of coordinates.

The action, reduced action, mass and angular momentum are therefore

$$
\begin{aligned}
S_{\mathrm{cl}}(T) & =-W=-\frac{T^{2}}{2 \pi \alpha^{\prime}}, \\
m & =\frac{T}{\pi \alpha^{\prime}}, \\
J & =\frac{T^{2}}{4 \pi^{2} \alpha^{\prime}},
\end{aligned}
$$

from which the relation follows

$$
\alpha^{\prime} m^{2}=4 J
$$

It should be noted that this relation differs from the standard one by a factor 4 . This is due to the different normalization of the string tension parameter $\alpha^{\prime}$. 


\section{B. Static Robertson-Walker spacetimes}

As a first curved spacetime, we examine the static Robertson-Walker universe, with line element

$$
\mathrm{d} s^{2}=-\mathrm{d} t^{2}+\frac{\mathrm{d} r^{2}}{1-\kappa r^{2}}+r^{2} \mathrm{~d} \phi^{2} .
$$

If $\kappa<0$, the potential

$$
\tilde{V}=\frac{\left(\frac{2 \pi r}{T}\right)^{2}-1}{1-\kappa r^{2}}
$$

is smaller than zero if $0<r<T / 2 \pi$, as in Minkowski spacetime. On the other hand, were we to take $\kappa>0$, the number of possible types of solutions increases. Consider first $\kappa<0$. Let $\nu=T \sqrt{-\kappa} / 2 \pi$, and $\mu=\nu / \sqrt{\nu^{2}+1}$.

Our computations result in

$$
\begin{aligned}
S_{\mathrm{cl}}(T) & =-\frac{4 T}{\pi \alpha^{\prime} \sqrt{-\kappa}} \frac{1}{\mu}[K(\mu)-E(\mu)], \\
W & =\frac{4 T}{\pi \alpha^{\prime} \sqrt{-\kappa}}\left[\frac{1}{\mu} E(\mu)+\frac{\mu^{2}-1}{\mu} K(\mu)\right], \\
m & =\frac{4}{\pi \alpha^{\prime} \sqrt{-\kappa}} \mu K(\mu),
\end{aligned}
$$

where $K$ and $E$ are complete elliptic integrals of the first and second kind respectively, with the elliptic modulus as their argument.

Let us now pass to the $\kappa>0$ situation. There are two classes of solutions: those that extend from 0 to $\min \left(r_{T}, r_{\kappa}\right)$, and those from $\max \left(r_{T}, r_{\kappa}\right)$ to infinity, where $r_{T}=T / 2 \pi$ and $r_{\kappa}=1 / \sqrt{\kappa}$. The second class of solutions lead to infinite reduced action. As to the first class, computations yield

$$
\begin{aligned}
S_{\mathrm{cl}}(T) & =-\frac{8 r_{\kappa}^{2}}{\alpha^{\prime}}\left[E\left(\frac{r_{T}}{r_{\kappa}}\right)+\left(\frac{r_{T}^{2}}{r_{\kappa}^{2}}-1\right) K\left(\frac{r_{T}}{r_{\kappa}}\right)\right], \\
W & =\frac{8 r_{\kappa}^{2}}{\alpha^{\prime}}\left[K\left(\frac{r_{T}}{r_{\kappa}}\right)-E\left(\frac{r_{T}}{r_{\kappa}}\right)\right], \\
m & =\frac{4 r_{T}}{\pi \alpha^{\prime}} K\left(\frac{r_{T}}{r_{\kappa}}\right),
\end{aligned}
$$

for the case $r_{T}<r_{\kappa}$, and

$$
\begin{aligned}
S_{\mathrm{cl}}(T) & =-\frac{8 r_{\kappa} r_{T}}{\alpha^{\prime}} E\left(\frac{r_{\kappa}}{r_{T}}\right), \\
W & =\frac{8 r_{\kappa} r_{T}}{\alpha^{\prime}}\left[K\left(\frac{r_{\kappa}}{r_{T}}\right)-E\left(\frac{r_{\kappa}}{r_{T}}\right)\right], \\
m & =\frac{4 r_{\kappa}}{\pi \alpha^{\prime}} K\left(\frac{r_{\kappa}}{r_{T}}\right),
\end{aligned}
$$

for the case $r_{T}>r_{\kappa}$. 
We see that the string angular momentum $J=W /(2 \pi)$ is not proportional to $m^{2}$ yielding a non-linear Regge trajectory.

In the $\kappa \rightarrow 0^{+}$limit, we have

$$
\begin{gathered}
W_{\stackrel{\kappa \rightarrow 0}{=}} \frac{T^{2}}{2 \pi \alpha^{\prime}}\left(1+\frac{3 T^{2}}{32 \pi^{2}} \kappa+\ldots\right), \\
m \stackrel{\kappa \rightarrow 0}{=} \frac{T}{\pi \alpha^{\prime}}\left(1+\frac{T^{2}}{16 \pi^{2}} \kappa+\ldots\right),
\end{gathered}
$$

and, consequently,

$$
\alpha^{\prime} m^{2} \stackrel{\kappa \rightarrow 0}{=} 4 n\left(1+\frac{n \alpha^{\prime}}{8} \kappa+\ldots\right)
$$

In the $\kappa \rightarrow 0^{+}$limit we find a linear Regge trajectory, recovering the previous results for Minkowski spacetime.

\section{Cosmological and black hole spacetimes}

Let us consider spacetimes with the generic form $g_{r r}=1 / a(r)=-1 / g_{t t}, g_{t \phi}=0$. The potential $\tilde{V}$ is then given by $\tilde{V}(r)=a(r)\left(\lambda^{2} g_{\phi \phi}-a(r)\right)$. Since the "motion" of $r$ in $\sigma$ can only take place when $\tilde{V}(r)<0$, we have to determine the zeroes of $a(r)$ and of $\lambda^{2} g_{\phi \phi}-a(r)$, together with the asymptotics in the different physical regions.

\section{1. de Sitter spacetime}

Included within this set of metrics we find the de Sitter metric, for which $a(r)=1-H^{2} r^{2}$ and $g_{\phi \phi}=r^{2}$. The radius of the horizon, $r_{H}$, is given by $r_{H}=1 / H$. Thus,

$$
\tilde{V}(r)=\left(1-H^{2} r^{2}\right)\left\{\left[H^{2}+\left(\frac{2 \pi}{T}\right)^{2}\right] r^{2}-1\right\} .
$$

The zeroes of the potential $V$ in this case are $r_{H}$ and $r_{H} / \sqrt{1+\left(\frac{2 \pi}{H T}\right)^{2}}$.

There are two types of planetoid strings: those of infinite length that are to be found outside the horizon, and those completely within the horizon, that are of finite length. Let us concentrate on the later. The maximum radius is $r_{\max }=r_{H} / \sqrt{1+(\lambda / H)^{2}}$ and $r_{\min }=0$. This is a string rotating around its middle point located precisely at $r=0$.

The integrals to be performed are complete elliptic integrals, with elliptic modulus

$$
k=H T / \sqrt{H^{2} T^{2}+4 \pi^{2}} .
$$

Let $k^{\prime}=\sqrt{1-k^{2}}$. Our computations result in the following:

$$
\begin{aligned}
S_{\mathrm{cl}}(T) & =-\frac{8}{k^{\prime} \alpha^{\prime} H^{2}}\left[E(k)-k^{2} K(k)\right], \\
W & =2 \pi J=\frac{8}{\alpha^{\prime} H^{2}} k^{\prime}[K(k)-E(k)],
\end{aligned}
$$




$$
m=\frac{4}{\pi H \alpha^{\prime}} k E(k)
$$

It is here obvious that $J$ is not proportional to $\mathrm{m}^{2}$.

For small $H T$, the quantization condition reads $T^{2} \sim 4 \pi^{2} n \alpha^{\prime}$, as in flat spacetime, and the mass of the string is in this case (compare with [9])

$$
\alpha^{\prime} m^{2} \simeq 4 n-7 H^{2} \alpha^{\prime} n^{2}+\cdots .
$$

It follows from eqs.(3.8) that $k$ is a two-valued function of $W$ and hence of $n$. Therefore, there are two values of $m$ for each $n$. This is easy to see from the behaviour of $W$ for $k \rightarrow 0$ and for $k \rightarrow 1$. $W$ vanishes in both cases.

$$
\begin{gathered}
W \stackrel{k \rightarrow 1}{=} \frac{8}{\alpha^{\prime} H^{2}} k^{\prime}\left(\log \frac{4}{k^{\prime}}-1\right)+O\left(k^{\prime 3} \log k^{\prime}\right), \\
W \stackrel{k \rightarrow 0}{=} \frac{2 \pi}{\alpha^{\prime} H^{2}} k^{2}+O\left(k^{4}\right) .
\end{gathered}
$$

There is a maximum on the values $n$ can take, given by

$$
n \leq n_{\max } \equiv 0.616 \frac{1}{\alpha^{\prime} H^{2}}
$$

This $n_{\max }$ correspond to the maximal planetoid mass.

The first branch yields masses in the range

$$
0 \leq m \leq m_{\max }=1.343 \ldots \frac{1}{H \alpha^{\prime}}
$$

and the second branch in the range

$$
\frac{4}{\pi \alpha^{\prime} H} \leq m \leq m_{\max }=1.343 \ldots \frac{1}{H \alpha^{\prime}} .
$$

[Notice that $\frac{4}{\pi}=1.2733 \ldots$. .

We find from eq.(2.6) for the invariant string length

$$
s=\frac{2}{H} \arcsin \frac{1}{\sqrt{1+\left(\frac{2 \pi}{H T}\right)^{2}}} .
$$

$s$ takes its maximum value $\frac{\pi}{H}$ for the lightest states in the second branch $k \rightarrow 1, m \rightarrow \frac{4}{\pi \alpha^{\prime} H}$. The shorter planetoids $s \simeq \frac{T}{\pi}, k \rightarrow 0$ correspond to the lightest states in the first branch.

With respect to the infinite length planetoid solutions (that is to say, those restricted to be outside the horizon), the corresponding action, reduced action and mass are all infinite. 


\section{Anti-de Sitter spacetime}

In this case, $a(r)=1+H^{2} r^{2}$ and $g_{\phi \phi}=r^{2}$. Only for a restricted set of values of $\lambda$ will there be a change of sign in $V$, since only if $\lambda^{2}>H^{2}$ will there be a zero of $V(r)$, namely at $1 / \sqrt{\lambda^{2}-H^{2}}$. Therefore, lower values of $\lambda$ correspond to strings of infinite length, whereas those strings for which $\lambda^{2}>H^{2}$ will be of finite length. They will rotate around its middle point located precisely at $r=0$ with period $T, 0<T<\frac{2 \pi}{H}$.

The results for this spacetime are as follows, where $k=H T /(2 \pi)=H / \lambda$ :

$$
\begin{aligned}
S_{\mathrm{cl}}(T) & =-\frac{8}{H^{2} \alpha^{\prime}}[K(k)-E(k)], \\
W & =\frac{8}{\left(H k^{\prime}\right)^{2} \alpha^{\prime}}\left[E(k)-\left(k^{\prime}\right)^{2} K(k)\right], \\
m & =\frac{4}{\pi H \alpha^{\prime}} \frac{k}{\left(k^{\prime}\right)^{2}} E(k) .
\end{aligned}
$$

In this case $W$ is a monotonous function of $T$, and so is $m$, so the doubling of mass eigenvalues found in de Sitter spacetime is not present here.

For the low-lying mass states we find

$$
\alpha^{\prime} m^{2} \simeq 4 n-H^{2} \alpha^{\prime} n^{2}+\cdots
$$

There is no upper bound in the mass spectrum for anti-de Sitter spacetime. For large masses we find

$$
m \simeq 2 n H, n>>1 .
$$

The heavy states spacing is given by $H$ whereas the small mass spacing is determined by $\left(\alpha^{\prime}\right)^{-\frac{1}{2}}$.

\section{Schwarzschild black hole}

For the Schwarzschild black hole $a(r)=1-2 M / r$ and $g_{\phi \phi}=r^{2}$, where $2 M$ stands for the Schwarzschild radius.

There will be positive zeroes of $V$ other than that at $2 M$ if and only if $16 \pi^{2} M^{2} / T^{2} \leq 4 / 27$. Of the two additional zeroes in this case, one will be placed between 1 and 1.5, and the other will be larger than 1.5 in units of $2 M$. In the extreme case $16 \pi^{2} M^{2} / T^{2}=4 / 27$ the two will coalesce onto $r_{0}=1.5$, which is the minimal (unstable) radius for a circular null geodesic [12]. As we turn $T$ to larger values, one of the zeroes runs to 1 , and the other out to infinity, these extreme values being reached for $2 \pi / T=0$, thus corresponding to an infinite static string from the horizon to infinity [13].

Let us choose the following parametrization for $T$, and consequently for the roots $r_{i}$ of $V$, with $r_{i}=2 M x_{i}$ :

$$
T=\frac{6 \pi M \sqrt{3}}{\cos (3 s)}
$$




$$
\begin{aligned}
& x_{1}=-\frac{3 \cos s}{\cos (3 s)}, \\
& x_{2}=\frac{3}{2 \cos (3 s)}(\cos s-\sqrt{3} \sin s), \\
& x_{3}=\frac{3}{2 \cos (3 s)}(\cos s+\sqrt{3} \sin s),
\end{aligned}
$$

whence $(r=2 M x)$

$$
\begin{aligned}
\tilde{V}(r) & =\frac{1}{x^{2}}(x-1)\left(\frac{4 \cos ^{2}(3 s) x^{3}}{27}-x+1\right) \\
& =\frac{4 \cos ^{2}(3 s)}{27 x^{2}}(x-1)\left(x-x_{3}\right)\left(x-x_{2}\right)\left(x-x_{1}\right) .
\end{aligned}
$$

The parameter $s$ is a function of $T / M$ as defined by eq.(3.12). $s$ runs from 0 to $\pi / 6$, and the roots are ordered as $x_{1}<1<x_{2}<x_{3}$. The planetoid string extends from $r=2 m x_{2}$ to $r=2 m x_{3}$. Its invariant length follows from eq.(2.4)

$$
s=2 M\left[f\left(x_{3}\right)-f\left(x_{2}\right)\right],
$$

where

$$
f(x)=\sqrt{x(x-1)}-\operatorname{ArgTh} \sqrt{1-\frac{1}{x}} .
$$

The classical, reduced action and mass are then integrals expressible in terms of elliptic integrals of modulus

$$
k^{2}=\frac{\left(x_{3}-x_{2}\right)\left(1-x_{1}\right)}{\left(x_{3}-1\right)\left(x_{2}-x_{1}\right)} .
$$

The explicit expressions are not by themselves very illuminating, since they involve combinations of elliptic integrals of different kinds; as a simple exponent, we have

$$
\begin{aligned}
m=\frac{T}{\pi^{2} \alpha^{\prime}} & \frac{2\left(x_{2}-1\right)}{\sqrt{\left(x_{3}-1\right)\left(x_{2}-x_{1}\right)}} \times \\
& \Pi\left(\frac{x_{3}-x_{2}}{x_{3}-1}, \sqrt{\frac{\left(x_{3}-x_{2}\right)\left(1-x_{1}\right)}{\left(x_{3}-1\right)\left(x_{2}-x_{1}\right)}}\right) .
\end{aligned}
$$

We use, as before, the notation of ref. [14].

An important point is that there is a minimum value for the reduced action and for the mass, corresponding to $T_{\min }=6 \pi \sqrt{3} M$, as follows:

$$
\begin{aligned}
& W_{\min }=\frac{36 \pi M^{2}}{\alpha^{\prime}}, \\
& m_{\min }=\frac{2 \sqrt{3} M}{\alpha^{\prime}} .
\end{aligned}
$$

The classical action for this configuration vanishes. 
The period $T$ has no upper bound. For large $T$ we find very long strings with

$$
W^{T \rightarrow \infty} \stackrel{1}{=} \frac{1}{6 \pi^{3} \alpha^{\prime} M} T^{3} \quad, \quad m \stackrel{T \rightarrow \infty}{=} \frac{1}{4 \pi^{3} \alpha^{\prime} M} T^{2} \quad, \quad s \stackrel{T \rightarrow \infty}{=} \frac{T}{2 \pi}-M \log \frac{T}{\pi M}+O(1)
$$

and the mass spectrum

$$
\left(\alpha^{\prime} M\right)^{1 / 3} m \stackrel{n \rightarrow \infty}{=}\left(\frac{9}{4 \pi}\right)^{1 / 3} n^{2 / 3}
$$

The Regge trajectory $W(m)$ is well behaved, and we portray it in Fig. 1.

\section{Schwarzschild black hole in de Sitter spacetime}

We shall now find competing effects due to the presence of one cosmological and one black hole horizons. The function $a(r)$ equals $1-2 M / r-H^{2} r^{2}$, and $g_{\phi \phi}=r^{2}$. We are presented with three cases:

- $1 /\left(27 M^{2}\right)>\left(4 \pi^{2} / T^{2}+H^{2}\right)$ (and, a fortiori, $\left.1 /\left(27 M^{2}\right)>H^{2}\right)$; the positive roots of the potential are the cosmological horizon, the black hole horizon, and two others, which we examine later.

- $\left(4 \pi^{2} / T^{2}+H^{2}\right)>1 /\left(27 M^{2}\right)>H^{2}$, when only strings inside the black hole horizon and outside the cosmological horizon are present within our ansatz.

- $H^{2}>1 /\left(27 M^{2}\right)$, which entails that there is no horizon and no strings of the form of our ansatz.

We shall now study the first of these cases, when there are four positive roots of the potential $V$, using a parametrization analogous to the one before. Let

$$
\begin{aligned}
x & =r /(2 M), \\
H & =\frac{\cos \left(3 s_{1}\right)}{3 \sqrt{3} M}, \\
T & =\frac{6 \sqrt{3} \pi M}{\sqrt{\cos ^{2}\left(3 s_{2}\right)-\cos ^{2}\left(3 s_{1}\right)}}, \\
x_{\mathrm{neg}} & =-\frac{3 \cos \left(s_{1}\right)}{\cos \left(3 s_{1}\right)}, \\
x_{S} & =\frac{3}{2 \cos \left(3 s_{1}\right)}\left(\cos \left(s_{1}\right)-\sqrt{3} \sin \left(s_{1}\right)\right), \\
x_{H} & =\frac{3}{2 \cos \left(3 s_{1}\right)}\left(\cos \left(s_{1}\right)+\sqrt{3} \sin \left(s_{1}\right)\right), \\
x_{\mathrm{nn}} & =-\frac{3 \cos \left(s_{2}\right)}{\cos \left(3 s_{2}\right)}, \\
x_{2} & =\frac{3}{2 \cos \left(3 s_{2}\right)}\left(\cos \left(s_{2}\right)-\sqrt{3} \sin \left(s_{2}\right)\right),
\end{aligned}
$$




$$
x_{3}=\frac{3}{2 \cos \left(3 s_{2}\right)}\left(\cos \left(s_{2}\right)+\sqrt{3} \sin \left(s_{2}\right)\right)
$$

with $0 \leq s_{2} \leq s_{1} \leq \pi / 6$. It follows that

$$
\begin{gathered}
\tilde{V}(r)=-\frac{16 \cos ^{2}\left(3 s_{1}\right) \cos ^{2}\left(3 s_{2}\right)}{729 x^{2}}\left(x-x_{\mathrm{neg}}\right)\left(x-x_{S}\right) \times \\
\left(x-x_{H}\right)\left(x-x_{\mathrm{nn}}\right)\left(x-x_{2}\right)\left(x-x_{3}\right) .
\end{gathered}
$$

Take $r_{i}=2 M x_{i}$. The four positive roots are ordered as follows: $r_{S} \leq r_{2} \leq r_{3} \leq r_{H}$. There are thus strings of the form of our ansatz extending from $r_{2}$ to $r_{3}$, and outside the cosmological horizon and inside the black hole horizon. The strings outside the cosmological horizon are of infinite length, mass and action. The really relevant ones for our purposes are those extending from $r_{2}$ to $r_{3}$, in complete analogy with the results for Schwarzschild's black hole. We portray a numerical computation of the classical Regge trajectory $W(m)$ in Fig. 2 for the case $s_{1}=\pi / 12$, that is, $H=\frac{1}{3} \sqrt{6} M$. Clearly to be seen are the two branches which had previously appeared for the rotating string in de Sitter spacetime. Surprisingly enough, there is no minimum value for $W$ and $m$ greater than zero in one of the branches, although it does appear in the second one. This is due to the numerical integration, which is very inexact in the limit $T \rightarrow T_{\min }(H)=6 \pi M \sqrt{3} / \sin \left(3 s_{1}\right)$, and the fact is that there is a minimum value for $W$, independent of $H$ and given by $W_{\min }=36 \pi M^{2} / \alpha^{\prime}$, as can be found by computing the adequate limit $s_{2} \rightarrow 0$; the mass $m$ also has a minimum value, but this time $H$ dependent: $m_{\min }=2 \sqrt{3} M \sin \left(3 s_{1}\right) / \alpha^{\prime}$. Notice that we recover the results previously obtained for Schwarzschild spacetime.

\section{CONCLUSIONS}

We have seen that the study of the planetoid solutions to the classical equations of motion of a string provides us with a variety of effects due to the structure of the target spacetime. In particular there are two main effects that we have uncovered:

1. the existence of a maximum value for the angular momentum of (equatorially) moving strings in spacetimes with particle horizons (de Sitter and Schwarzschild-de Sitter in particular), which reflects itself on the existence of two branches in the Regge plot. This means that the number of bound states is finite in the semiclassical quantization. (But this finiteness must be exact, beyond the semiclassical approximation).

2. the presence of a minimum value for the angular momentum in the case of a black hole event horizon, as in Schwarzschild and Schwarzschild-de Sitter spacetimes.

It is not difficult to understand this phenomenon in the light of elementary quantum mechanics. In spacetimes with particle horizons it is necessary for the preservation of causality that if a string extends beyond the horizon that it be infinite. The length is quantized in the same manner that the angular momentum is, as can be read out from eq.(2.5); it is thus the case that there are a finite number of possible quantum planetoid strings.

As to the minimum value, given that if a string does penetrate into a (Schwarzschild) event horizon and is to maintain its linearity it must extend to infinity, we see that the 
"cutting out" of part of the spacetimes is what forces a minimum value even for classical values (quantum mechanically that was only to be expected).

String solutions that generalize non-circular point particle trajectories should also exist

in the spacetimes considered here. However, the $\sigma$ and $\tau$ dependence probably cannot be separated as we did in the planetoid strings presented in this paper.

We want to stress that the Regge trajectories are no longer linear (even for weak curvature) in the spacetimes considered here. We thus infer from this classical test string calculations that the fundamental string spectrum will get strongly modified in these non-trivial gravitational backgrounds.

\section{ACKNOWLEDGMENTS}

ILE has to thank the LPTHE for their hospitality on several occasions. 


\section{Figure Captions}

Fig.1: The reduced action $W=2 \pi J$ (where $J$ is the angular momentum) in units of $\pi M^{2} / \alpha^{\prime}$ as a function of the string mass $m$ in Schwarzschild spacetime.

Fig. 2: The reduced action $W=2 \pi J$ (where $J$ is the angular momentum) as a function of the string mass $m$ in Schwarzschild-de Sitter spacetime. 


\section{REFERENCES}

$\dagger \quad$ e-mail: wtpegegi@lg.ehu.es

[1] H. J. de Vega and N. Sánchez Phys. Lett. B 197 320, (1987).

H. J. de Vega and N. Sánchez, Nucl. Phys. B 309, 552 and 577 (1988).

[2] H. J. de Vega and N. Sánchez in Proceedings of the Erice Schools: "String Quantum Gravity and Physics at the Planck Energy Scale", 21-28 June 1992, Edited by N. Sánchez, World Scientific, 1993, and Third D.Chalonge School, 4-16 September 1994.

[3] H.J. de Vega and N. Sánchez in the Proceedings of the D. Chalonge School, 1995, N. Sánchez and A. Zichichi, editors, Kluwer 1996.

[4] F. Combes, H. J. de Vega, A. V. Mikhailov and N. Sánchez, Phys. Rev. D50, 2754 (1994).

I. Krichever, Funct. Anal. and Appl. 28, 21 (1994),

[5] P.S. Letelier, P.R. Holvorcem and G. Grebot, Class. Quantum Grav. 7, 597-610 (1990).

[6] H.J. de Vega and I.L. Egusquiza, Phys. Rev. D49 763-778 (1994).

[7] A.L. Larsen and N. Sánchez, Phys. Rev. D50 7493-7518 (1994).

[8] A.L. Larsen and N. Sánchez, Phys. Rev. D51 6929-6948 (1995).

[9] H. J. de Vega, A.L. Larsen, and N. Sánchez, Phys. Rev. D 51 6917, (1995).

[10] A.L. Larsen and N. Sánchez, "Sinh-Gordon, Cosh-Gordon and Liouville Equations for Strings and Multi-Strings in Constant Curvature Spacetimes", hep-th/96003049.

[11] W.G. Dixon, Proc. Roy. Soc. Lond. A 314 499-527 (1970).

[12] S. Chandrasekhar, The Mathematical Theory of Black Holes, Oxford University Press, New York (1992).

[13] V.P. Frolov, V.D. Skarzhinsky, A.I. Zelnikov and O. Heinrich, Phys. Lett. B 224225 (1989).

[14] I.S. Gradshteyn and I.M. Ryzhik, Table of Integrals, Series, and Products, Academic Press, London (1990). 